\title{
Status of the STEREO experiment
}

\section{Stephane Zsoldos*†}

Laboratoire de Physique Subatomique et Cosmologie, Grenoble, France, CNRS/IN2P3/UGA

E-mail: zsoldoselpsc.in2p3.fr

The re-evaluation of the theoretical antineutrino flux emitted by nuclear reactors revealed a deficit of about $6 \%$ between the observed flux and the expected one. This so-called reactor antineutrino anomaly has a statistical significance of $2.7 \sigma$, and one possible explanation to this deficit could be the existence of a light sterile neutrino state into which reactor antineutrino oscillate at very short distances. The STEREO project, which will be presented in this talk, aims to find an evidence of such oscillation.

The measurement will take place at only few meters $(9 \mathrm{~m}-11 \mathrm{~m})$ from the compact core of the Institut Laue-Langevin research reactor in Grenoble (France), which provides a large flux of electron antineutrinos with an energy range from 1 to $10 \mathrm{MeV}$. The sensitive volume of the STEREO detector is $2 \mathrm{~m}^{3}$ of organic liquid scintillator doped with Gadolinium, consisting of 6 cells stacked along the direction of the core and detecting antineutrinos via inverse- $\beta$ decay.

This setup will provide excellent sensitivity to short-baseline oscillations effects by precisely measuring any relative distortion of the antineutrino spectrum as a function of both energy and baseline.

Close proximity to the reactor yield a high background environment that is managed through heavy shielding surrounding the detector. A water-cerenkov muon veto will be settled above the detector to tag incoming cosmic muons.

The detector is currently under construction and is expected to deliver its first results in spring 2017.

38th International Conference on High Energy Physics 3-10 August 2016

Chicago, USA

${ }^{*}$ Speaker.

${ }^{\dagger}$ At Queen Mary University London since October 2016. 


\section{A search for light sterile neutrinos}

\subsection{The Reactor Antineutrino Anomaly}

In 2011, the reactor group at CEA-IRFU in Saclay, France, performed a reevalution of the antineutrino flux spectrum emitted by nuclear reactors in preparation for the Double CHOOZ experiment. The prediction of the antineutrino flux is based on the measurement of the $\beta$-spectrum at the Institut Laue-Langevin (ILL) research reactor in Grenoble (France) during the 1980's which is converted afterwards into antineutrino [1] [2]. By taking in consideration non-equilibrium effect on some nuclei during the fission of the ILL nuclear reactor fuel, they have shown that the flux is 3\% higher that previously computed [3]. This results lead to the reanalysis of the past reactor antineutrino measurements. These previous experiences were detecting antineutrino through the inverse $\beta$-decay process, $\bar{v}_{e}+p \rightarrow e^{+}+n$, whose cross-section is inversely proportional to the neutron lifetime [4]. In addition with the recent measurements of a reduce neutron lifetime compared to the previous one [5], the combination of these results lead to the so-called Reactor Antineutrino Anomaly (RAA), which indicates a $2.9 \sigma$ deficit from the flux expected [6]. Moreover, an other anomaly discovered previously while calibrating solar neutrino detector with intense $\beta^{+}$sources shows a deficit which is compatible with the reactor antineutrino anomaly [7]. One possible interpretation of this deficit could be the existence of a light sterile neutrino state into which reactor antineutrino oscillates at very short distances [8]. The mixing angle and squared mass splitting of this new neutrino state can be computed using the neutrino oscillation framework by adding a new dimension to the PMNS neutrino mixing matrix. The reanalysis point for best fit parameters values at $\sin ^{2} 2 \theta_{s}=0.17$ and $\Delta m_{s}^{2}=2.1 \mathrm{eV}$ [8], which is three orders of magnitude heavier than the known actives neutrino states.
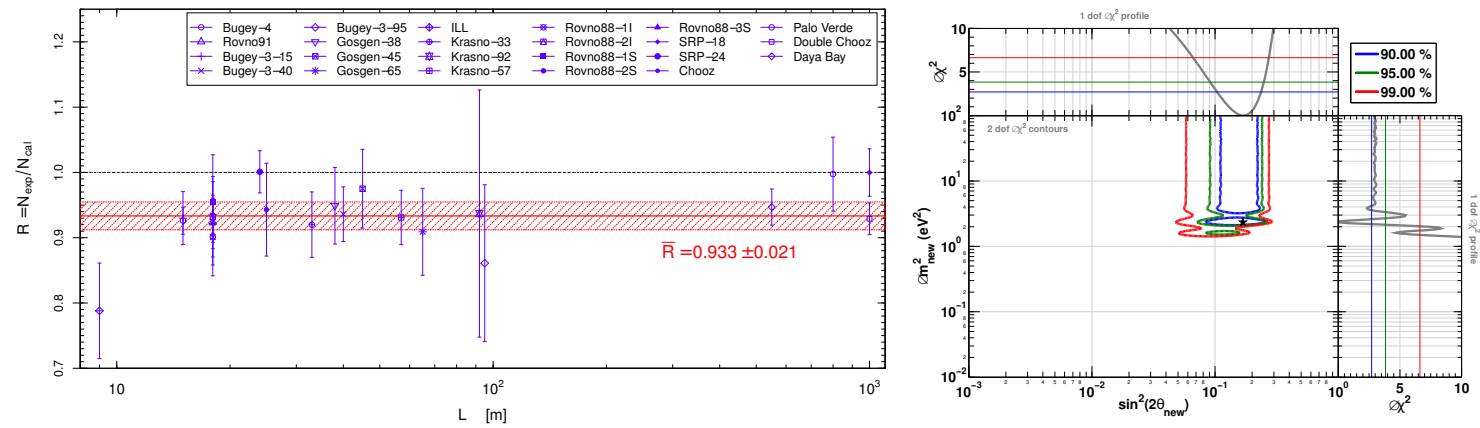

Figure 1: Left : Ratio of neutrinos detected over expected at several distances from the neutrino source. Points represent measurements made by previous experiments and the blue line shows the 3-neutrinos oscillation model prevision [9]. Right : Phase space $\Delta m_{s}^{2}$ vs $\sin ^{2} 2 \theta_{s}$ allowed for a new sterile oscillation compatible with the RAA [8].

\subsection{Up-to-date on experiments at low $\Delta m^{2}$}

At the moment, a huge effort is produce by the neutrino scientific community to measure the neutrino flux at very short distances from an intense emitter, like nuclear reactors or highlyactive ${ }^{144} \mathrm{Ce}$ radioactive sources. The neutrinos emitted range between [1-10] $\mathrm{MeV}$, and using 
the neutrino oscillation probability formula, one can compute the distance $L_{s}$ where the oscillation should occur, for the parameter $\Delta m_{s}^{2} \approx \mathrm{eV}$ :

$$
L_{s}[\mathbf{m}] \equiv \frac{E[\mathbf{M e V}]}{1.27 \times \Delta m_{s}^{2}[\mathbf{e V}]}=\frac{[1-10] \mathbf{M e V}}{1.27 \times[1] \mathbf{e V}} \approx[1-10] \mathbf{m}
$$

Table 1 shows the few experiments running and about to aiming at measuring the antineutrino flux at close distance from a nuclear reactor. The reactor experiments are all based on the inverse- $\beta$ decay reaction for antineutrinos detection in a liquid or solid scintillator, doped with a neutronabsorbing metal like gadolinium, lithium or boron. The experimental signature of the inverse- $\beta$ decay process is based on a delayed coincidence between the positron annihilation and the neutron capture.

\begin{tabular}{l|c|c|c|c|c|c|c|c}
\hline \hline Name & $\begin{array}{c}\mathrm{P}_{t h} \\
(\mathrm{MW})\end{array}$ & $\begin{array}{c}\mathrm{L} \\
(\mathrm{m})\end{array}$ & $\begin{array}{c}\text { Overburden } \\
(\mathrm{m} . w . e)\end{array}$ & $\begin{array}{c}\mathrm{M}_{\text {target }} \\
(\text { Tons })\end{array}$ & Tech & Seg & Mov & Exp start \\
\hline \hline Nucifer & 70 & 7 & 13 & 0.8 & $\mathrm{Gd}$ & $\mathrm{x}$ & $\mathrm{x}$ & 2014 \\
\hline NEOS far & 2800 & 25 & $16-23$ & 1 & $\mathrm{Gd}$ & $\mathrm{x}$ & $\checkmark$ & 2016 \\
\hline NEOS near & 15 & 5 & 23 & 1 & $\mathrm{Gd}$ & $\mathrm{x}$ & $\checkmark$ & 2017 \\
\hline STEREO & 57 & $9-11$ & 18 & 1.75 & $\mathrm{Gd}$ & $\checkmark$ & $\checkmark$ & 2016 \\
\hline SoLi $\delta$ & 70 & $5.5-10$ & 10 & 2.9 & ${ }^{6} \mathrm{Li}$ & $\checkmark$ & $\checkmark$ & 2016 \\
\hline DANSS & 3000 & $9.7-12.2$ & 50 & 0.9 & $\mathrm{Gd}$ & $\checkmark$ & $\checkmark$ & 2016 \\
\hline Neutrino-4 & 100 & $6-12$ & 10 & 1.5 & $\mathrm{Gd}$ & $\mathrm{x}$ & $\checkmark$ & 2016 \\
\hline Prospect & 85 & $7-18$ & few & $1-10$ & $\mathrm{Gd}+{ }^{6} \mathrm{Li}$ & $\checkmark$ & $\checkmark$ & 2017 \\
\hline NuLat & 85 & $3-8$ & few & 1 & ${ }^{6} \mathrm{Li}+{ }^{10} \mathrm{~B}$ & $\checkmark$ & $\checkmark$ & 2017 \\
\hline
\end{tabular}

Table 1: Table of future reactor neutrino experiments aiming at probing the reactor antineutrino anomaly and the sterile neutrino hypothesis at $\Delta m_{s}^{2} \approx 1 \mathrm{eV}$. We indicated the thermal power of the nuclear reactor, the overburden in meter water equivalent, the fiducial mass of detection in tons, the technology of the detector based on liquid or solid scintillator and with an additional dopant to enhance neutron capture. Segmentation of the detector and possibility to move it from the reactor core are also shown.

\section{The STEREO experiment}

The STEREO experiment [10] aims at measuring the rate and energy spectrum of antineutrinos emitted by the ILL reactor in Grenoble, France, at six different positions between $9 \mathrm{~m}$ and $11 \mathrm{~m}$ from the core.

Antineutrinos are detected through their inverse $\beta$-decay reaction in a liquid scintillator doped with $0.2 \%$ of gadolinium. This concentration will delayed the neutron capture from the positron annihilation by $15 \mu \mathrm{s}$, allowing to sign the reaction and reduce drastically the contribution of accidental background events. Antineutrinos energy will be reconstructed thanks to the energy deposition of the positron in the liquid scintillator plus its annihilation with an electron. Considering the target protons at rest, and neglecting the neutron kinetic energy of the order of $20 \mathrm{keV}$, we defined the antineutrino energy with respect to the visible energy from the positron annihilation by : 


$$
\begin{aligned}
E_{v i s} & =E_{e^{+}}+E_{e^{-}} \\
& =E_{\bar{v}_{e}}-\left(m_{n}-m_{p}\right)+m_{e^{-}} \\
& =E_{\bar{v}_{e}}-0.782 \mathbf{M e V}
\end{aligned}
$$

Figure 2 shows the expected antineutrino spectrum at $9 \mathrm{~m}$ and at $11 \mathrm{~m}$ from the ILL core in the presence of a sterile oscillation at the best fit parameters and without. The energy resolution is crucial to be able to distinguish between both cases.
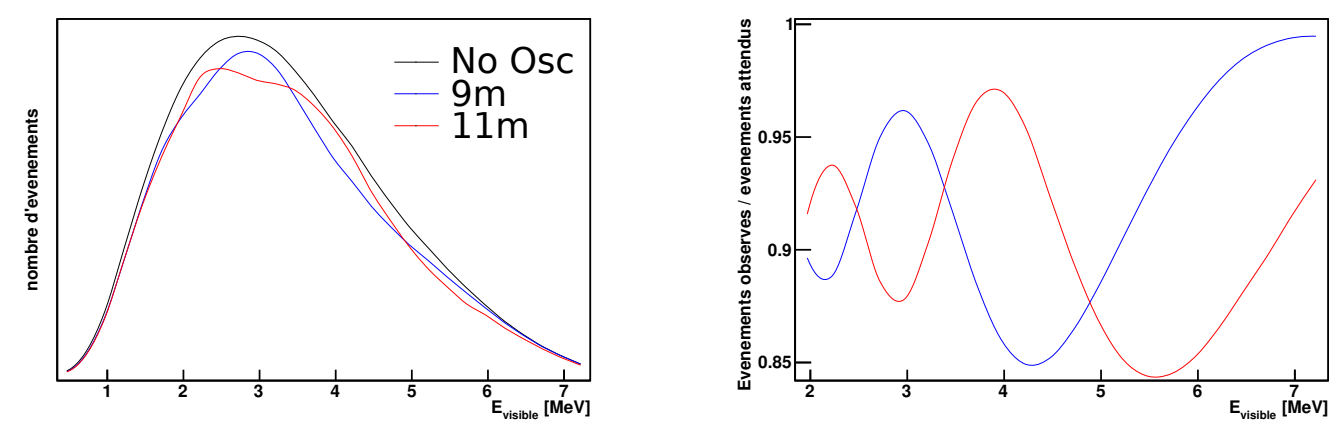

Figure 2: Left : Expected spectrum without sterile oscillation at $\Delta m_{s}^{2} \approx 1 \mathrm{eV}$ (black line) at a close distance from the core $(<100 \mathrm{~m}$ ), and at $9 \mathrm{~m}$ (blue line) and $11 \mathrm{~m}$ (red line) in the case of an actual sterile oscillation at $\Delta m_{s}^{2} \approx 1 \mathrm{eV}$. Right : Oscillation probability vs neutrino energy at $9 \mathrm{~m}$ from source (blue line) and at $11 \mathrm{~m}$ from source (red line) in the case of the sterile neutrino oscillation at $\Delta m_{s}^{2} \approx 1 \mathrm{eV}$.

Close proximity to the nuclear core will also lead to a huge background environment in neutrons and gamma rays, which will be moderated by using heavy passive shielding of polyethylene and lead to thermalize neutrons and absorb gamma rays. On the top of STEREO a water-Cerenkov detector will be set up and act as a muon veto for the antineutrino selection in the data. Muons will produce numerous amount of fast neutron during their journey through matter and especially through STEREO's lead shielding, and these fast neutrons can produce a correlated background by mimicking the prompt signal through a proton recoil of the liquid scintillator before being thermalized and capture on a gadolinium nucleus.

\section{The STEREO detector}

The STEREO detector contains a fiducial volume of 1.75 tons of liquid scintillator divided into six cells stacked along the way of the neutrino flux (defined as the $\vec{z}$ axis on Figure 3 ) provided by the ILL research nuclear reactor. Cells are identical and measure $89 \times 91 \times 37 \mathrm{~cm}^{3}$. The ILL nuclear reactor core consists of a compact cylinder of highly enriched uranium 235 (93\%) of $40 \mathrm{~cm}$ diameter and $80 \mathrm{~cm}$ tall. The barycentre of the fuel remains constant during a working cycle of 50 days thanks to an automated control system and using neutrons poisons like boron. The neutrino flux is then expected to be constant during the reactor ON period. The dimension of the cell in the $\vec{z}$ direction define the vertex resolution for recontruction, which is about the dimension of the ILL core. 


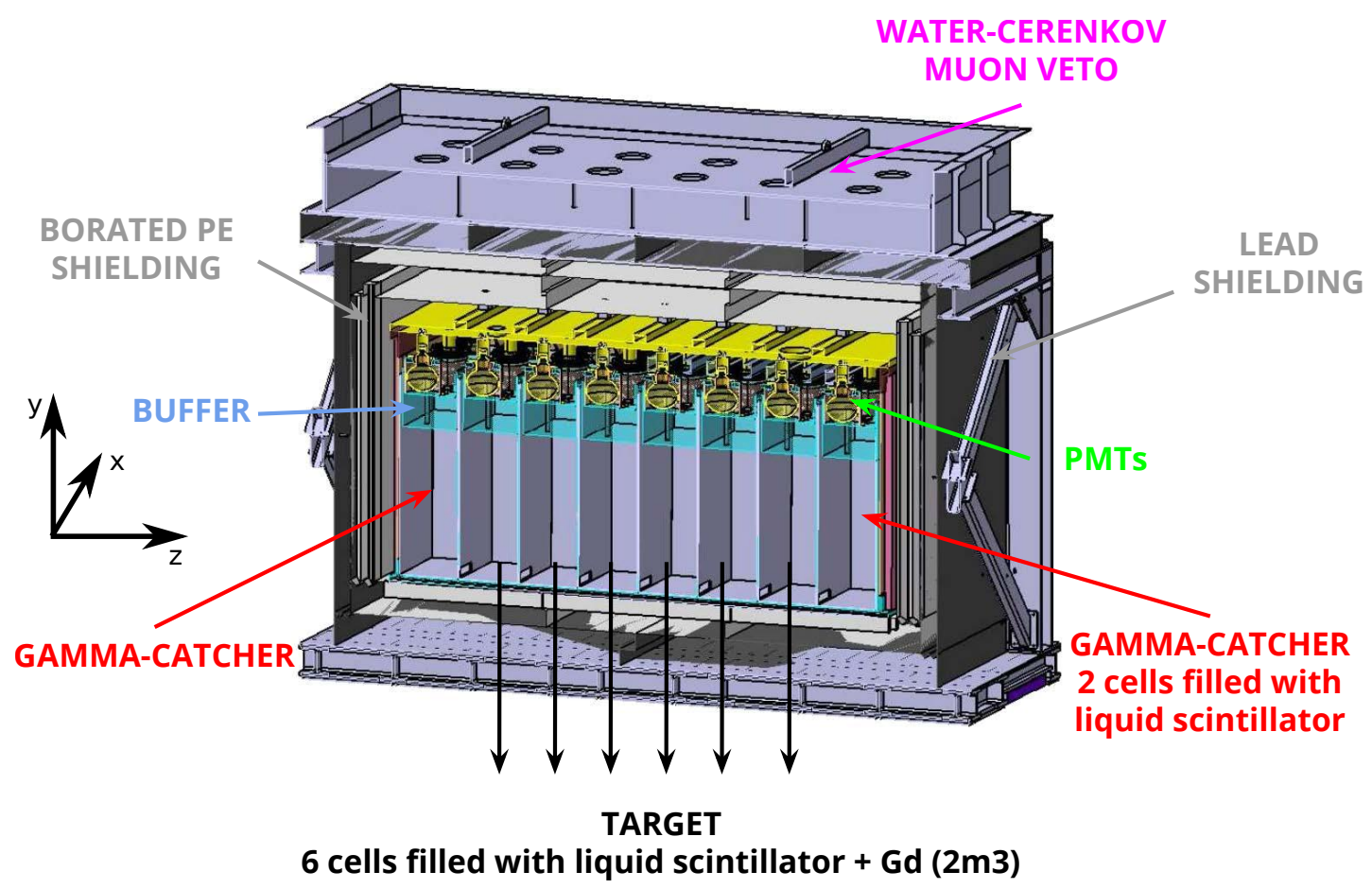

Figure 3: Scheme of the STEREO detector

4 Hamamatsu R5912-100 PMT's are installed above each cells and are separated from the liquid scintillator by an acrylic buffer filled with a mineral oil. This setup allows to reduce asymetries of light collection between events produced at the top or bottom of the cells, and materials have similar optical indices assuring the light coupling to the PMT's.

Surrounding the fiducial volume of detection defined by the six cells is set an active crown consisting of segments filled with liquid scintillator but without gadolinium, covered by Hamamatsu R5912-100 on the top (4 PMTs for segments along the $\vec{z}$ axis, 8 for lateral segments). This crown is called the gamma-catcher and aims at collect any gamma rays produce by the annihiliation of a positron which could escape the volume defined by the cells, and likewise enhance the energy resolution. Simulation with $2 \mathrm{MeV}$ positrons estimated an energy resolution of $12 \%$ with small differences between cells.

The detector is covered by $10 \mathrm{~cm}$ of lead shieldings in every direction with an extra $20 \mathrm{~cm}$ underneath, and $15 \mathrm{~cm}$ of polyethylene on the side, $20 \mathrm{~cm}$ above and $30 \mathrm{~cm}$ on the top. Moreover, soft iron plates and a cage of $\mu$-metal surrounds the STEREO detector to shield the PMT's from magnetic fields produced by nearby experiments. Background measurements campaign have been extensively performed on site to design the passive shieldings and construction has also been undertaken to add lead and polyethylene shieldings to the walls of the ILL site where STEREO is installed.

On the top of STEREO is installed a water-Cerenkov detector which aims at tagging muon event in the analysis to define a muon veto to antineutrino selection. The muon veto consist of a stainless steel tank covered with tyvek sheets and filled with $2500 \mathrm{~L}$ of water. 20 Hamamatsu 
R5912-100 PMT's are installed above the detector and facing downwards. A wavelength shifter is added to the water to enhance Cerenkov light collection by the PMTs. Design and a dedicated trigger system as been defined to achieve a measured $99.3 \%$ detection efficiency to muons whereas the gamma efficiency if less than $0.5 \%$.

A dedicated electronics had been developped for the STEREO detector [11] which is hosted on a $\mu$ TCA crate. Front-end boards are in charge of first level triggering and acquisition, by using 8-channel FADC 14 bits continuously sampling at $250 \mathrm{MHz}$. These boards compute the charge of the signal, the charge of the tail of the signal and the time at a constant fraction of the signal. A specific trigger board is in charge of the second level programmable trigger thanks to a FPGA by taking into account the different parts of the detector, i.e the cells, the gamma-catcher and the muon veto. A last board is in charge of the light calibration of the detector using a LED driver.

Calibration of the detector is made by using 3 subsystems which will transport radioactives sources around the detector (measuring the energy scale), under (study neutron capture efficiency), and also a manual subsytem allowing to insert sources inside tubes in 3 cells -2 central and one border.

\section{Sensitivity}

The expected sensitivity of the experiment is shown in Figure 4. The STEREO detector will be able to cover all the reactor antineutrino anomaly in 2 years of data taking with a 95\%CL. Detection and reconstruction of systematics are included, as well as systematics of the antineutrino spectrum.

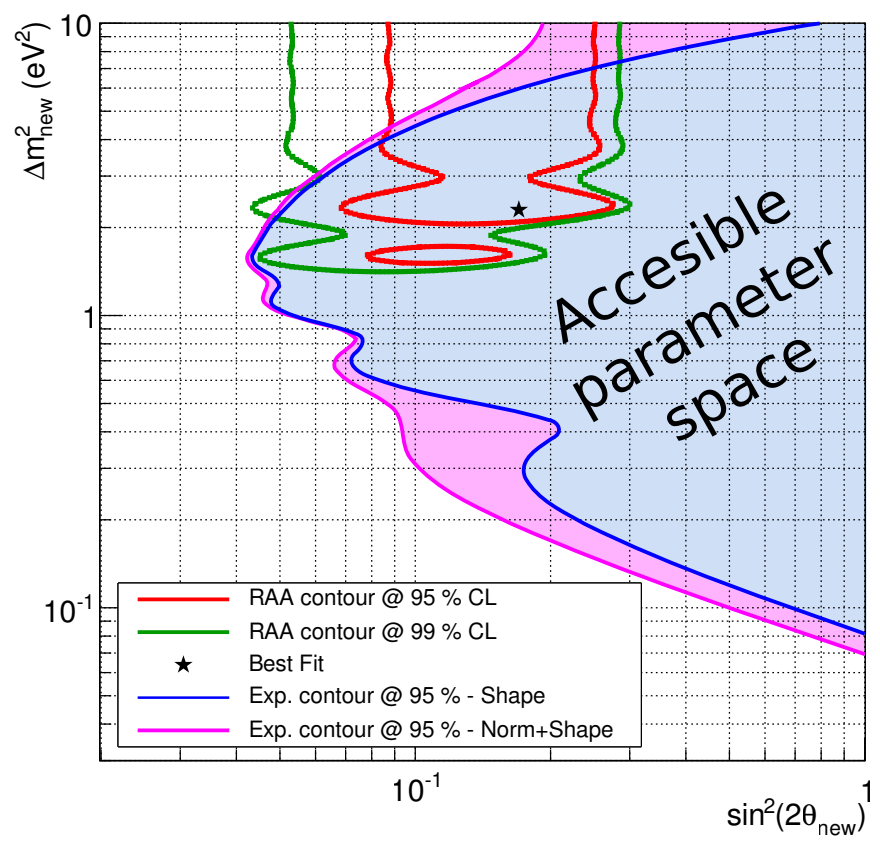

Figure 4: Sensitivity of the STEREO experiment. Area at the right of the blue and pink contours are the region explored by the detector after 300 days of data taking at $95 \% \mathrm{CL}$, respectively with the energy shape distorsion analysis only and with the shape + norm analysis. The red and green contours are the region allowed by the RAA at 95\% CL and 99\% CL respectively, and the star shows the best fit parameters. 


\section{References}

[1] K. Schreckenbach, "Absolute Measurement of the Beta Spectrum from 235U Fission as a Basis for Reactor Antineutrino Experiment." Physics Letters B 99, no. 3 (1981).

[2] K. Schreckenbach, "Determination of the Antineutrino Spectrum from 235U Thermal Neutron Fission Products up to 9.5MeV." Physics Letters B 160, no. 4,5 (1985).

[3] Th. A. Mueller, "Improved Predictions of Reactor Antineutrino Spectra." Physical Review C 83, 54615 (2011).

[4] P. Vogel and J. F. Beacom, Phys. Rev. D60 (4), 053003 (1999).

[5] C. Patrignani et al. (Particle Data Group), Chin. Phys. C, 40, 100001 (2016).

[6] G. Mention, M. Fechner, Th. Lasserre, Th. A. Mueller, D. Lhuillier, M. Cribier, and A. Letourneau. "The Reactor Antineutrino Anomaly." Physical Review D 83, 7 (April 29, 2011). doi:10.1103/PhysRevD.83.073006.

[7] C. Giunti and M. Laveder, "Statistical Significance of the Gallium Anomaly," Physical Review D 83, 065504 (2011) doi:10.1103/PhysRevC.83.065504

[8] K.N. Abazajian and others, "Light Sterile Neutrinos: A White Paper." arXiv:1204.5379

[9] C. Giunti, "Oscillations beyond three-neutrino mixing", Neutrino 2016

[10] D. Lhuillier and others, "Proposal of a Search for Sterile Neutrinos at ILL : The STEREO Experiment," 2012.

[11] O. Bourrion et al., "Trigger and readout electronics for the STEREO experiment", JINST 11, no. 02, C02078 (2016) doi:10.1088/1748-0221/11/02/C02078 [arXiv:1510.08238 [physics.ins-det]]. 